\title{
Limit Cycle Oscillation Suppression Controller Design and Stability Analysis of the Periodically Time-varying Flapping Flight Dynamics in Hover
}

\section{Liang Wang}

Beihang University School of Automation Science and Electrical Engineering

\section{Wuyao Jiang}

Beihang University School of Automation Science and Electrical Engineering

\section{Zongxia Jiao}

Beihang University School of Automation Science and Electrical Engineering

Longfei Zhao ( $\square$ zhaolf@buaa.edu.cn )

Beihang University https://orcid.org/0000-0002-0044-5267

\section{Research Article}

Keywords: Wing-tail interaction, Periodically time-varying dynamics, Poincaré map, Floquet theory, Active disturbance rejection controller

Posted Date: August 19th, 2021

DOl: https://doi.org/10.21203/rs.3.rs-802587/v1

License: (c) (i) This work is licensed under a Creative Commons Attribution 4.0 International License. Read Full License

Version of Record: A version of this preprint was published at Nonlinear Dynamics on January 15th, 2022. See the published version at https://doi.org/10.1007/s11071-021-07145-0. 


\title{
Limit cycle oscillation suppression controller design and stability analysis of the periodically time-varying flapping flight dynamics in hover
}

\author{
Liang Wang $\cdot$ Wuyao Jiang $\cdot$ Zongxia Jiao $\cdot$ Longfei Zhao
}

\begin{abstract}
The periodically time-varying forces make the equilibrium state of Beihawk, an X-shaped flapping-wing aircraft, to be a periodic limit cycle oscillation. However, traditional controllers based on averaging theory fail to suppress this oscillation and the derived stability result may be inaccurate. In this study, a period-based method is proposed to design the oscillation suppression controller, locate the corresponding cycle and analyze its stability. A periodically time-varying wing-tail interaction model is built and Discrete Fourier Transform is applied to adapt the model for controller design. The harmonics less than quintuple flapping frequency account for more than 96 percent of the total harmonics and are reserved to present a concise model. Based on this model, Active Disturbance Rejection Controller (ADRC) is designed and its Extended State Observer can observe the disturbance to suppress the oscillation. Poincaré map is introduced to convert the stability analysis of the cycle to a fixed point. A multiple shooting method is adopted to locate several points on the cycle and
\end{abstract}

L. Wang $\cdot$ W. Jiang $\cdot$ Z. Jiao

School of Automation Science and Electrical Engineering, Beihang University, Beijing 100191, China

L. Wang $\cdot$ W. Jiang $\cdot$ Z. Jiao $\cdot$ L. Zhao $(\bowtie)$

Science and Technology on Aircraft Control Laboratory, Beihang University, Beijing 100191, China

e-mail: zhaolf@buaa.edu.cn

Z. Jiao $\cdot$ L. Zhao

Ningbo Institute of Technology, Beihang University, Ningbo 315800, China

L. Zhao

Research Institute for Frontier Science, Beihang University, 100191, Beijing, China 
the map is obtained by calculating the submaps between the adjacent points with the Floquet theory. The located points are proved to be accurate compared with the numerical solved cycle and the stability analysis result of the cycle is verified by the dynamic evolution. Compared with the State Feedback Controller, the ADRC performs better in suppressing the limit cycle oscillation and eliminating the attitude control error. The oscillation suppression is meaningful in maintaining a stable flight and capturing high quality images.

Keywords: Wing-tail interaction $\cdot$ Periodically time-varying dynamics $\cdot$ Poincaré map $\cdot$ Floquet theory $\cdot$ Active disturbance rejection controller

\section{Introduction}

The flapping-wing aircraft equipped with good maneuverability, fine flexibility and high flight efficiency is designed based on the motivation of flying creatures like birds and insects, and has become a hot area of research recently [1, 2]. However, the constant dynamic oscillation resulting from their inherent flapping propulsion mechanism makes it difficult to hover steadily like a rotorcraft. And this will be more serious in large aircraft because of the scale effect and thus existing hoverable flapping-wing aircraft are mostly insect-like [3-5]. So, this paper challenges the oscillation suppression control of a large flapping-wing aircraft, Beihawk weighed 1.2 $\mathrm{kg}$ with $1.5 \mathrm{~m}$ wingspan [6], to enable a stable hover.

The hover maneuverability of flapping-wing aircraft can be attributed to their unsteady high-lift mechanisms which cannot be explained by traditional steady aerodynamic theories for fixed-wing aircraft due to their low Reynolds number regime and unsteady characteristics [7]. Great achievements have been made in the unsteady aerodynamic study of flapping flight in the past decades [8-11], among which, Dickinson et al. [9] summarized these mechanisms as delayed stall, rotational lift, added mass and wake capture, etc. They investigated the aerodynamic force generation of a fruit fly by using a dynamically scaled robotic model and various instantaneous force measuring experiments were conducted. The obtained force data was fitted by mathematical empirical formulas to approximate these unsteady mechanisms. Owing to the high precision and intuitive analytical expression for the unsteady mechanisms, it has been widely used in the optimization of wing kinematics [12], dynamic modelling [13] and controller design [14]. 
The solved aerodynamic forces in flapping-wing aircraft are periodically timevarying and they are embedded into the dynamic equations of motion to constitute a periodically time-varying model. Such a model is a typical non-autonomous system with heavy reliance on time and it is thus hard to design controllers and analyze the stability [15]. To address it, the assumption that the flapping frequency is much larger than the body's natural frequency is introduced and averaging theory is thus applied to simplify such a model into a time-averaged (autonomous) one [16]. Averaging theory bridges the gap of controller design and stability analysis between the flappingwing and fixed-wing aircraft and Deng et al. conducted a series of flapping flight control studies with this foundation. Linear quadratic gaussian optimal controller [16], nonlinear controllers including a neural adaptive controller [17], a geometric controller [18] and a reinforcement learning control policy [19] were separately designed. Basic attitude stability augmentation [17] and trajectory tracking [16, 20] were firstly achieved, and then some extreme maneuvers [19] could be performed with the aid of these controllers. Wood et al. [21] adopted this theory as well and adaptive controllers were designed to cope with the uncertainties arisen from manufacturing imperfections in flapping flight with great success. Both Deng's and Wood's aircraft are micro vehicles with high flapping frequency and the body dynamics is mainly affected by the averaged forces. While for large ones, the frequency is strictly limited by the large inertial force of the wing and the averaging operation may be invalid. Although great achievements have been made under the averaging theory, there still exist some debates about the valid range of this theory [22]. Besides, averaging theory averages the inherent dynamic oscillation in flapping flight and controllers designed based on the averaged model behave badly in oscillation suppression.

Even more, the typical time-invariant controller stability analysis methods such as the Lyapunov method and Eigenvalue method based on the averaged model may fail to evaluate the practical periodic stability of flapping flight. This is because the equilibrium state of flapping flight is no longer a point but a periodic orbit. This orbit should not be averaged since only by including this periodic orbit can we explain the large attitude oscillations and the stall phenomena at some extreme instantaneous flapping positions in large flapping-wing aircraft. Many efforts have been done to consider this periodic property in flapping flight as fully as possible. Taylor et al. [23] 
firstly built a periodically time-varying flapping flight model of locust and redefined the stability issues as the asymptotic limit cycle stability in the phase space. Their work has been considered to be the most comprehensive and realistic description of flapping flight dynamics. Su et al. [24] followed this approach by building an approximate linear periodically time-varying model of a flapping-wing aircraft and performed the stability analysis based on the transition matrix in Floquet theory. Nevertheless, this method is strictly confined by the linear model assumption. Dietl et al. [25] further improved this approach by introducing a shooting algorithm to search for the trimmed limit cycle of the open-loop dynamics and proved its instability. Yet they did not design controllers to stabilize the aircraft due to the constraints of the model complexity. In summary, present periodic studies are not overall and systematic. They either concentrated on periodic dynamic modelling, or tried to evaluate the inherent periodic stability of the flapping creatures or the open-loop flapping-wing aircraft. And to date, there is no such research which exploits the related periodic theories to solve the practical control problem in flapping-wing aircraft to obtain a stable limit cycle and minimize its oscillation as far as possible.

The periodic issues are especially evident for the pitching attitude of the target aircraft in hover, so it is rare for such a large flapping-wing aircraft as Beihawk to hover. The main issues can be concluded as two aspects. First, in addition to the periodic thrust force for weight balance in hover, the $\mathrm{X}$-shaped flapping wing also generates some periodic disturbing torque which would exacerbate the attitude oscillation. Second, the flapping wing generates thrust force through accelerating the surrounding air and a strong induced flow is generated. This flow is the source of the inflow over the tail in hover stage and possesses the same periodic time-varying principle as the thrust force, which would reduce the tail control effect in oscillation suppression. The previous work [6] has modelled this flow and the disturbance, and designed simple PD controller to validate this model. The designed controller could maintain the aircraft in hover and the pitching attitude oscillation was not further analyzed. Indeed, these oscillatory behaviours inevitably degrade the quality of images obtained and cause frequent malfunctions in mounted onboard microelectronics that are vulnerable to external vibrations. Therefore, it is necessary to build a both accurate enough and easily analyzed periodic model, design periodic controllers to suppress the oscillation based on this model and prove the stability of 
the controllers from the periodic limit cycle viewpoint.

The objective of this paper is to investigate an easily implemented periodically time-varying model and design an efficient limit cycle suppression controller with the wing-tail interaction model. In particular, the stability of the designed controller is analyzed by a period-based method to include the unique limit cycle in flapping flight. A pitching attitude model of Beihawk including unsteady effects is firstly presented. Specially the flapping-wing induced flow over the tail is considered to give a wingtail interaction model. Discrete Fourier Transform (DFT) is performed on the aerodynamic forces and the significant harmonic contents are incorporated into the dynamic model to present a concise model. The model-free State Feedback Controller (SFC) and the model-based Active Disturbance Rejection Controller (ADRC) are separately designed to suppress the oscillation. The stability of the controller is proved by the stability of the corresponding equilibrium limit cycle. A multiple shooting method based on Levenberg-Marquardt Algorithm (LMA) is utilized to locate the cycle and its stability is described with the characteristic multipliers by introducing the Poincaré map and Floquet theory.

The rest of this paper is organized as follows. Section 2 gives the periodically timevarying wing-tail interaction attitude model of Beihawk. Section 3 describes the DFT of the model and the attitude controller design. Section 4 reports the period-based stability analysis method of the designed controllers. Section 5 presents the limit cycle oscillation suppression effects. Section 6 summarizes the conclusions and proposes some potential applications.

\section{Attitude dynamic model of Beihawk}

The wing aerodynamics, tail aerodynamics and body dynamics form the model of Beihawk. Three significant high-lift mechanisms which are delayed stall, rotational lift and added mass are considered in the wing model. The rotation of the wing manipulates not only the magnitude of the aerodynamic forces but also the orientation [26]. However, the averaged rotational lift equals to zero [7], which also indicates the drawback of the averaging theory and the necessity of a periodically time-varying model. The wing generates thrust force through accelerating the momentum of the surrounding air and thus momentum theory [27] is adopted to relate the induced flow velocity to the thrust force. This flow is the main incoming flow over the tail in hover 
and a periodic tail model including the wing induced flow is thus established. The wing model and tail model are incorporated into the dynamic model to give the final periodically time-varying model.

\subsection{Wing aerodynamics including unsteady effects}

The blade element theory with the aerodynamic coefficients identified from experiment is adopted to approximate the unsteady effects.

The flapping angle $\phi$ and twisting angle $\beta$ are given by

$$
\left\{\begin{array}{l}
\phi(t)=\phi_{\max } \cos (2 \pi f t) \\
\beta(t)=\arctan \left(\frac{n}{N} \tan \left(\beta_{\max }\right)\right) \sin (2 \pi f t)
\end{array}(n=1, \ldots, N) .\right.
$$

In a quasi-steady model, the force equations derived from thin aerofoils translating with constant velocity and angle of attack also apply to time-varying flapping wings [13]. Therefore, the translational aerodynamic forces exerted on per blade element aerofoil are given by

$$
\begin{aligned}
& d F_{t r, N}(t, r)=\frac{1}{2} C_{N}(\alpha(t)) \rho c(r) U^{2}(t, r) d r, \\
& d F_{t r, T}(t, r)=\frac{1}{2} C_{T}(\alpha(t)) \rho c(r) U^{2}(t, r) d r,
\end{aligned}
$$

where $d F_{t r, N}$ and $d F_{t r, T}$ separately represent the normal force and tangential force of the wing. Dickinson et al. [28] solved the force coefficients due to delayed stall as follows,

$$
\begin{aligned}
& C_{N}(\alpha)=3.4 \sin \alpha \\
& C_{T}(\alpha)= \begin{cases}0.4 \cos ^{2}(2 \alpha), & 0 \leq \alpha \leq 45^{\circ} . \\
0, & \text { otherwise }\end{cases}
\end{aligned}
$$

Fung et al. [29] derived the theoretical force exerted on per blade element aerofoil due to rotational lift as

$$
d F_{r o t, N}(t, r)=\frac{1}{2} C_{r o t} \rho c(r)^{2} U(t, r) \omega d r,
$$

where $C_{r o t}=2 \pi\left(0.75-\hat{x}_{0}\right)$ is the rotational coefficient, $\hat{x}_{0}$ is the dimensionless distance of the longitudinal rotation axis from the leading edge, $\omega$ is the instantaneous angular velocity of the wing, approximating to $\dot{\alpha}(t)$.

In [30], an additional normal force contributed by the added mass effect is given by

$$
d F_{a d d, N}(t, r)=\frac{\rho \pi c^{2}(r)}{4} \dot{v}_{2} d r
$$


where $\dot{v}_{2}$ is the normal acceleration at the mid chord due to the wing motion.

The total normal force and tangential force exerted on per blade element aerofoil are given by,

$$
\begin{gathered}
d F_{N}(t, r)=d F_{t r, N}(t, r)+d F_{r o t, N}(t, r)+d F_{a d d, N}(t, r), \\
d F_{T}(t, r)=d F_{t r, T}(t, r) .
\end{gathered}
$$

These forces are rotated into body axis by

$$
\begin{aligned}
& d N(t, r)=d F_{N}(t, r) \cos \beta+d F_{T}(t, r) \sin \beta \\
& d T(t, r)=d F_{T}(t, r) \cos \beta+d F_{N}(t, r) \sin \beta
\end{aligned} .
$$

The up-wing and down-wing are different due to the design of the dihedral angle $\phi_{0}$ (see Fig. 1). Therefore, the flapping and pitching motions of the up-wing and down-wing can be listed as follows:

$$
\left\{\begin{array}{l}
\phi_{\text {up }}(t)=\phi_{\max } \cos (2 \pi f t)-\phi_{0} \\
\phi_{\text {down }}(t)=-\phi_{\max } \cos (2 \pi f t)-\phi_{0} \\
\beta_{\text {up }}(t)=\arctan \left(\frac{n}{N} \tan \left(\beta_{\max }\right)\right) \sin (2 \pi f t) \\
\beta_{\text {down }}(t)=-\arctan \left(\frac{n}{N} \tan \left(\beta_{\max }\right)\right) \sin (2 \pi f t)
\end{array} .\right.
$$

The whole wings' instantaneous normal and thrust forces are obtained by integration as follows,

$$
\begin{aligned}
& N(t)=2 \int_{0}^{b / 2} \cos \phi_{u p} d N(t, r)_{u p}+\cos \phi_{d o w n} d N(t, r)_{d o w n} . \\
& T(t)=2 \int_{0}^{b / 2} d T(t, r)_{u p}+d T(t, r)_{d o w n}
\end{aligned}
$$

The detailed introduction to the layout and description of the parameters of Beihawk can refer to the previous work [6].

\subsection{Tail aerodynamics considering wing-tail interaction}

Momentum theory [27], modelling the circular swept area of the flapping wing as an actuator disk that accelerates the surrounding air, is used to calculate the flapping wing induced flow velocity over the tail. The momentum change of the induced flow generates the thrust force and its velocity at the wing root is given by

$$
V_{i}(0)=0.5 \sqrt{\frac{T(t)}{S_{d}} \frac{1}{2 \rho}},
$$

where $T(\mathrm{t})$ is the thrust force. This flow changes along the body and the velocity at $x$ (see Fig. 1 for axis definition) position is given by 


$$
V_{i}(x)=V_{i}(0)\left[1+\frac{x / R}{\sqrt{1+(x / R)^{2}}}\right],
$$

where $R$ is the diameter of the actuator disk, i.e., b/2. Beihawk's tail is installed approximately $0.5 \mathrm{~m}$ from the wing root, and the induced flow velocity at the tail, i.e., $V_{i T}$, can then be obtained.

Bernoulli's equation is used to calculate the aerodynamic force acting on the tail as follows,

$$
F_{T}(t)=\frac{1}{2} \rho V_{i T}^{2} S_{\text {tail }} C_{l}^{\alpha} \delta,
$$

where $\delta$ is the tail pitch angle. With thin flat-plate-airfoil assumption, the lift-curve slope of a finite span three dimensional wing is given by [31]

$$
C_{l}^{\alpha}=\frac{2 \pi}{1+(2 \pi / \pi \sqcap A R)(1+\tau)},
$$

where $A R$ is the aspect ratio, and $\tau$ is 0.04 for the designed wing [32].

\subsection{Periodically time-varying model of Beihawk}

The pitch torque generated by the wing can be calculated based on the centre of pressure (CP) of the wing, $A_{W}$,

$$
M_{W}(t)=N(t) \llbracket x_{W}^{0}-T(t) \llbracket z_{W}^{0} .
$$

Considering the fact that the flapping amplitude is only $15 \mathrm{deg}$, the position variation of $\mathrm{CP}$ is not significant. Therefore, the $\mathrm{CP}$ position of each flapping wing is assumed to be located at the average flap plane of each flapping wing, and at $1 / 4 \mathrm{c}$ position along the chord direction. The overall $\mathrm{CP}$ of a pair of flapping wings is then decided at the middle of the two $\mathrm{CP}$ point of each flapping wing.

The tail torque is calculated based on the $\mathrm{CP}$ of the tail, i.e., $A_{T}$,

$$
M_{T}(t)=F_{T}(t) \square x_{T}^{0},
$$

where $x_{T}^{0}$ is the distance between $A_{T}$ and $G$ along the $x$ axis. 


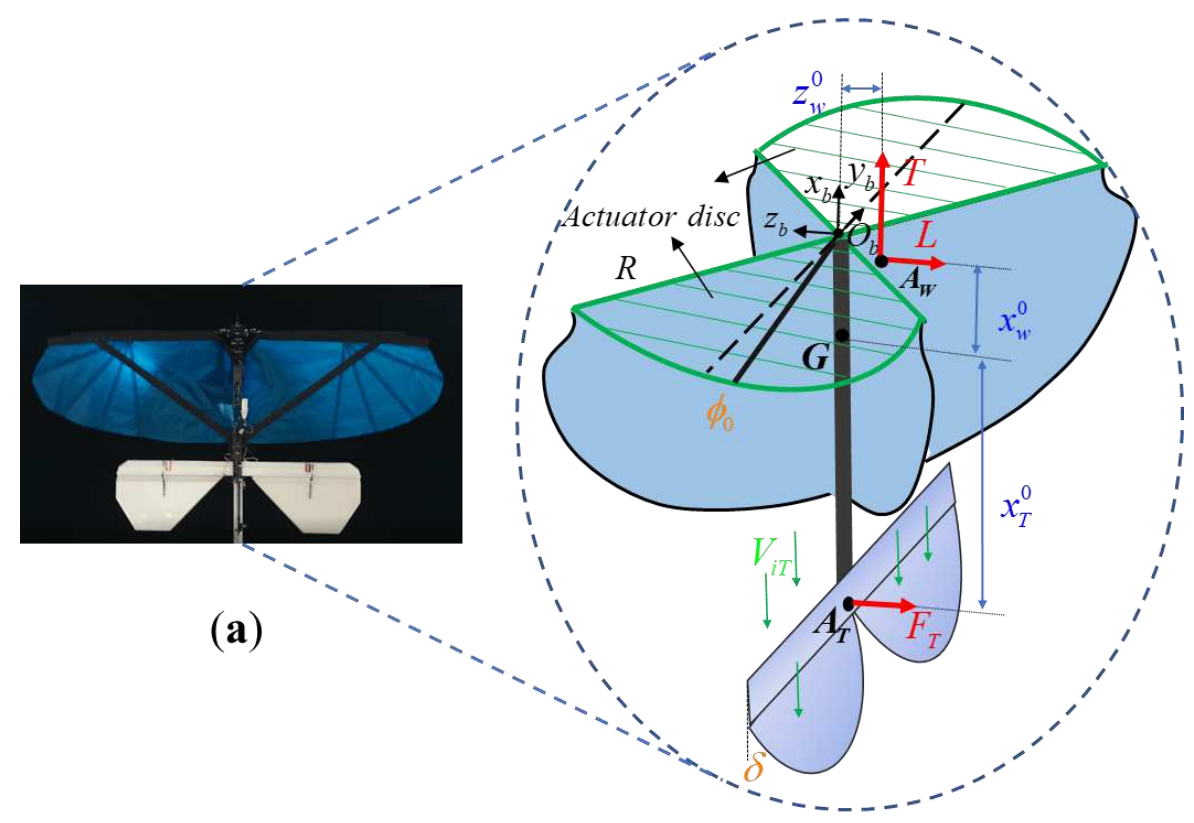

(b)

Fig. 1 (a) Practical image of Beihawk, (b) Beihawk prototype schematic (Including coordinates and parameters definition).

The longitudinal damping of Beihawk is much smaller than the lateral and thus hovering stability is mainly affected by the pitching attitude dynamics which is given by

$$
\begin{aligned}
& \dot{\theta}(t)=q(t) \\
& \dot{q}(t)=\frac{1}{I_{y y}} N(t) \llbracket x_{W}^{0}-\frac{1}{I_{y y}} T(t) \llbracket z_{W}^{0}+\frac{1}{I_{y y}} F_{T}(t) \llbracket x_{T}^{0} .
\end{aligned}
$$

The controlled variable is tail pitch angle and Eq. (18) can be further summarized as follows according to Eq. (14)

$$
\begin{aligned}
& \dot{\theta}(t)=q(t) \\
& \dot{q}(t)=\frac{1}{I_{y y}} N(t) \llbracket x_{W}^{0}-\frac{1}{I_{y y}} T(t) \llbracket z_{W}^{0}+\frac{1}{I_{y y}} 0.3 \square T(t) \square \delta
\end{aligned}
$$

It is obvious that Eq. (19) is periodically time-varying under the periodically forced terms $N(t)$ and $T(t)$. The tail torque is influenced by the thrust force generated by the wings, and thus wing-tail interaction is fully considered. Beihawk relies on the tail to suppress the attitude oscillation by counteracting the disturbing torque generated by flapping wings. Whereas the tail torque possesses the same periodic time-variation principle as the thrust force, which would reduce the tail control effect if we still adopt traditional controllers based on constant incoming flow over the tail. 


\section{Attitude controller design}

The complex aerodynamic formulas in flapping flight make it impossible to give a concise aerodynamic model which can be easily embedded into the dynamic model to constitute an appropriate model for controller design. Therefore, present researchers either directly control the generation of the aerodynamic forces by skipping the aerodynamic model, or simplify the complex model by employing averaging theory. Apparently, both assumptions neglect the most important unsteady aerodynamic features.

In this section, we adapt the model built in Section 2 to controller design by taking Discrete Fourier Transform (DFT) to the instantaneous aerodynamic forces. The transformed force is composed of infinite discrete fourier series: the fundamental harmonic frequency is the same as the flapping frequency, the direct component corresponds to the averaged value, and the harmonics far above the body's natural frequency will not cause subtle changes on the body dynamics and thus can be neglected. The above operation makes the original model convenient for controller design and stability analysis, and reserves the periodically time-varying features as well.

Based on the transformed model, two controllers are designed. The model-free State Feedback Controller (SFC) is designed to primarily achieve the stability augmentation of the hover maneuver. Advanced model-based controller, Active Disturbance Rejection Controller (ADRC) is further designed to suppress the oscillation. The Extended State Observer (ESO) in ADRC can make full use of the built periodic wing-tail interaction model to observe not only the model state but also the disturbance as the extended state. The ESO has been proved to be efficient in disturbance observation and the observed disturbance can then be counteracted in the control signal design.

\subsection{Main fourier series representation of the model}

The normal force and thrust force generated by the flapping motion are functions of the periodic wing kinematics and can thus be presented by the fourier terms, i.e., 


$$
\begin{aligned}
& F(t)=\sum_{n=0}^{h}\left[a_{n} \cos (2 \pi f n t)+b_{n} \sin (2 \pi f n t)\right], \\
& c_{n}=\sqrt{a_{n}^{2}+b_{n}^{2}}
\end{aligned}
$$

where $n$ is an integer and $\mathrm{f}$ is the flapping frequency. Any non-harmonic spectral content can be treated as noise. Set the hover flapping frequency as $8 \mathrm{~Hz}$ and we can obtain the corresponding aerodynamic forces over one whole flapping period. One thousand uniformly distributed data points in a period are selected for DFT and the results are presented below,

(a)

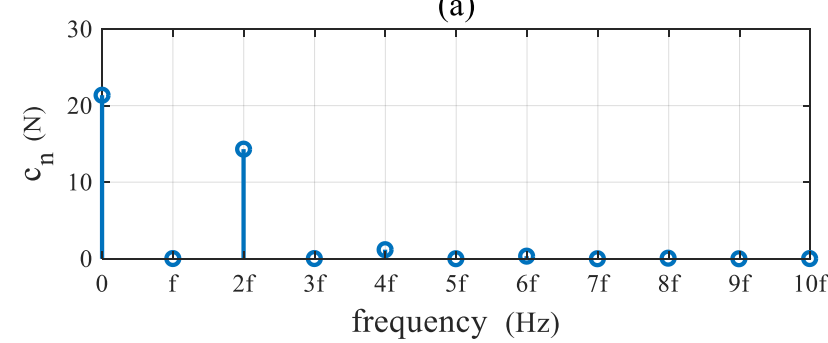

(b)

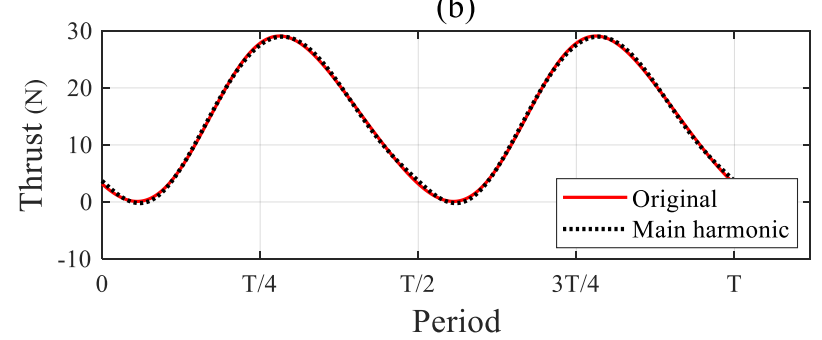

Fig. 2 DFT result of the thrust force: (a) Amplitude vs frequency characteristic diagram, (b) The comparison between the original thrust force and the thrust force represented by the main harmonic.

The amplitude-frequency characteristic diagram (Fig. 2(a)) indicates that the harmonics below $4 \mathrm{f} \mathrm{Hz}$ (include) are the main harmonic content of the thrust force. Besides, the harmonic above $4 \mathrm{f} \mathrm{Hz}$ is much higher than the body's natural frequency and the body dynamics is mainly influenced by the averaged value. The contribution of these harmonics can be overlooked, since the averaged value equals zero. Therefore, the main harmonics are chosen to approximate the original thrust force and the result is shown in Fig. 2(b), it is nearly the same as the original force. 
(a)

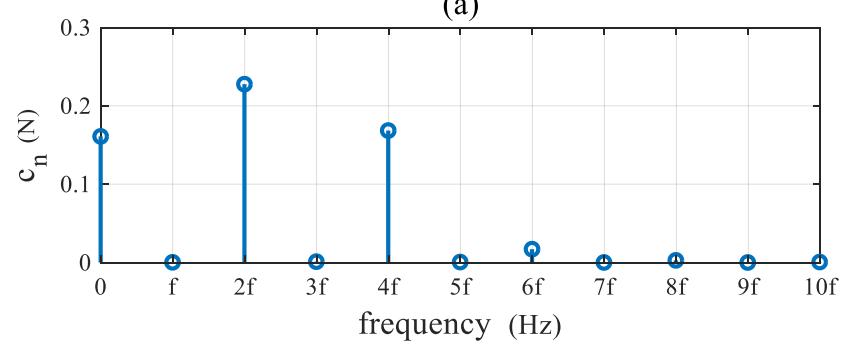

(b)

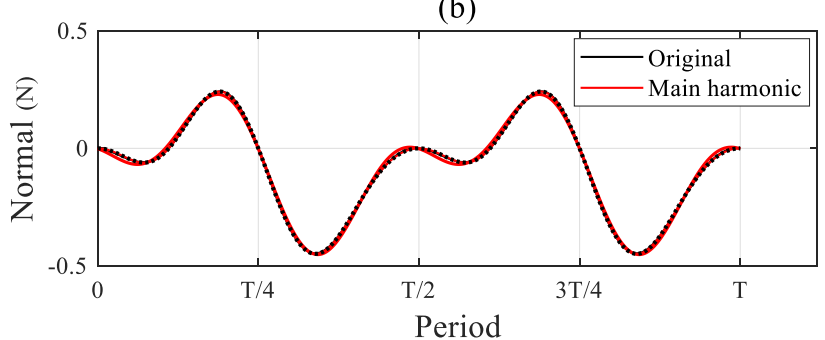

Fig. 3 DFT result of the normal force: (a) Amplitude vs frequency characteristic diagram, (b) The comparison between the original normal force and the normal force represented by the main harmonics.

The same operation is carried out for the normal force and the result is shown in Fig. 3. We also select harmonics below $4 \mathrm{f} \mathrm{Hz}$ (include) as the main harmonic content of the normal force. The transformed thrust force and normal force are represented as

$$
\begin{aligned}
T_{h}(t) & =\sum_{n=0}^{4}\left[a_{T n} \cos (2 \pi f n t)+b_{T n} \sin (2 \pi f n t)\right], \\
N_{h}(t) & =\sum_{n=0}^{4}\left[a_{N n} \cos (2 \pi f n t)+b_{N n} \sin (2 \pi f n t)\right] .
\end{aligned}
$$

The percentage of each harmonic content in the thrust force and normal force is calculated separately and the results are listed in Table 1 . It can be easily seen that the harmonics below $4 \mathrm{f} \mathrm{Hz}$ (include) accounts for more than $98 \%$ of the whole harmonics in the thrust force and $96 \%$ in the normal force, which also proves the rationality of our approximation.

Table 1 The percentage of each harmonic content in the thrust and normal force

\begin{tabular}{ccccccccccc}
\hline Harmonic & 0f & 1f & 2f & 3f & 4f & 5f & 6f & 7f & 8f & 9f \\
\hline$T(\%)$ & 57.13 & 0.08 & 38.28 & 0.08 & 3.17 & 0.02 & 0.91 & 0.01 & 0.21 & 0.01 \\
\hline$N(\%)$ & 27.78 & 0.05 & 39.27 & 0.19 & 29.06 & 0.10 & 2.94 & 0.02 & 0.48 & 0.01 \\
\hline
\end{tabular}

The main fourier series representation of the Eq. (19) is written as

$$
\begin{aligned}
& \dot{\theta}(t)=q(t) \\
& \dot{q}(t)=\frac{1}{I_{y y}} N_{h}(t) \llbracket x_{W}^{0}-\frac{1}{I_{y y}} T_{h}(t) \llbracket z_{W}^{0}+\frac{1}{I_{y y}} 0.3 \square T_{h}(t) \square \delta .
\end{aligned}
$$


Suppose $\quad x_{1}=\theta(t), \quad x_{2}=q(t), \quad d(t)=\frac{1}{I_{y y}} N_{h}(t) \llbracket x_{W}^{0}-\frac{1}{I_{y y}} T_{h}(t) \llbracket z_{W}^{0}, \quad b(t)=\frac{1}{I_{y y}} 0.3 \llbracket T_{h}(t)$ and $u(t)=\delta$, Eq. (23) can be rewritten as,

$$
\begin{aligned}
& \dot{x}_{1}(t)=x_{2}(t) \\
& \dot{x}_{2}(t)=d(t)+b(t) u(t)
\end{aligned}
$$

Eq. (24) is also a non-autonomous system, or more specifically a periodically timevarying system and the built wing-tail interaction model is reflected in $b(t)$, which is a time-varying term along with the periodic flapping motion.

\subsection{Limit cycle oscillation suppression controller design}

In this section, two controllers are separately designed to suppress the attitude oscillation of Beihawk, the basic SFC and the advanced ADRC.

The SFC is designed as follows,

$$
\begin{aligned}
& \dot{x}_{1}=x_{2} \\
& \dot{x}_{2}=d(t)+b(t)\left[k_{p}\left(x_{1 d}-x_{1}\right)+k_{d}\left(x_{2 d}-x_{2}\right),\right.
\end{aligned}
$$

where $x_{1 d}$ and $x_{2 d}$ are separately the expected value of $x_{1}$ and $x_{2}, k_{p}$ and $k_{d}$ are the corresponding feedback gains.

SFC is simply designed without including model information. It can only achieve the stability augmentation and cannot well reject the disturbing torque generated by the wings. The tail control effect is noticeably reduced under this traditional SFC because of the time-varying induced flow. Therefore, it is urgent to design modelbased controllers to take full advantage of the built model.

ADRC was first proposed by Han [33] and has been widely used in the industrial control [34] and quadrotor control [35] due to its excellent disturbance rejection capacity. It is employed here with the following superiorities. First, it can take full advantage of the wing-tail interaction model built in section 2.2 to achieve more accurate disturbance observation and better disturbance rejection. Second, it can observe the pitching attitude velocity and thus the attitude velocity sensors can be replaced, which would reduce the weight of the aircraft and prolong the endurance. The ADRC is implemented as follows.

In fact, in the context of feedback control, $d(t)$ in Eq. (24) is something to be overcome by the control signal. Treating $d(t)$ as an additional state variable, $x_{3}=d(t)$, and let $\dot{d}(t)=G(t)$ with $G(t)$ unknown, the original plant Eq. (24) is 
now described as

$$
\begin{aligned}
& \dot{x}_{1}(t)=x_{2}(t) \\
& \dot{x}_{2}(t)=x_{3}(t)+b(t) u(t), \\
& \dot{x}_{3}(t)=G(t)
\end{aligned}
$$

which is always observable. Then, the periodic ESO can be constructed as follows,

$$
\begin{aligned}
& \dot{\hat{x}}_{1}=\hat{x}_{2}+l_{1}\left(x_{1}-\hat{x}_{1}\right) \\
& \dot{\hat{x}}_{2}=\hat{x}_{3}+b(t) u+l_{2}\left(x_{1}-\hat{x}_{1}\right), \\
& \dot{\hat{x}}_{3}=l_{3}\left(x_{1}-\hat{x}_{1}\right)
\end{aligned}
$$

where the system output $x_{1}$ and the control signal $u$ are the inputs to ESO, and the output of the ESO, $\hat{x}_{1}, \hat{x}_{2}$ and $\hat{x}_{3}$ are respectively the observation of $x_{1}, x_{2}$ and $d(t) . l_{1}, l_{2}$ and $l_{3}$ are the gains of the observer. There is no doubt that better observation results can be obtained with the help of the built tail model $b(t)$.

Note that the important information $d(t)$, provided by the ESO, allows the control law $u=\left(u_{0}-d(t)\right) / b(t)$ to reduce the plant in Eq. (24) to a cascade integral form of

$$
\begin{aligned}
& \dot{x}_{1}(t)=x_{2}(t) \\
& \dot{x}_{2}(t)=u_{0}
\end{aligned}
$$

Therefore, the ADRC can utilize the built wing-tail interaction model to compensate the plant and the compensated model can be easily controlled by making $u_{0}$ a function of the tracking error and its derivative, i.e., a SFC. Based on the above description, the periodic control signal of the ADRC in our paper is designed as

$$
u=\frac{1}{b(t)}\left[k_{1}\left(x_{1 d}-x_{1}\right)+k_{2}\left(x_{2 d}-\hat{x}_{2}\right)-\hat{x}_{3}\right] .
$$

\section{Stability analysis of the designed controller}

The designed controllers in Section 3 are also periodically time-varying since it is based on the periodically time-varying model. Considering this periodically timevarying feature, there is no equilibrium state where all states remain constant as the equilibrium point in fixed-wing aircraft. Therefore, general stability analysis methods for fixed-wing aircraft cannot be directly utilized to evaluate the stability of flappingwing aircraft.

In this section, a period-based method is proposed to analyze the stability of the 
controller. The model including the controller can be written as a periodically timevarying system,

$$
\dot{x}=f(t, x),(t, x) \in R^{1} \times R^{n},
$$

where $f\left(t+T_{0}, x\right)=f(t, x)$ and $T_{0}$ is the period. The equilibrium state of the system is a periodic orbit and is defined as a limit cycle. The stability of the system can be proved by proving the stability of the limit cycle. The defined limit cycle is stable if any trajectory starting near the limit cycle converges on it. If any trajectory starting near the limit cycle diverges from it, the limit cycle is unstable.

Our method converts the stability of a limit cycle to the stability of a fixed point in the hyperplane by introducing the Poincaré map. In an autonomous system, the stability of the fixed point is determined by the characteristic multipliers of the Poincaré map's Jacobian matrix. This conclusion is further generalized to the periodically time-varying system, i.e., a special non-autonomous system by defining a specific Poincaré map. The corresponding theorem is presented and also proved in this paper.

To solve the characteristic multipliers of the original system, a variation equation of the cycle is introduced and the system is transformed into a linear periodically timevarying system. Floquet theory applied for linear periodically time-varying system is utilized to solve the monodromy matrix and this matrix can be regarded as a Poincaré map. Floquet multipliers of this matrix are thus proved to be coincident with the multipliers of the aforementioned map. Finally, the key theorem on the stability of the periodically time-varying flapping flight system is given.

The exact location of the limit cycle is prerequisite for the monodromy matrix. A multiple shooting method is introduced to locate the limit cycle by shooting several points on it. Levenberg-Marquardt Algorithm (LMA) is adopted to iterate these solutions until these points converge on the circle. Two adjacent points can be related by the transition matrix and all these transition matrixes are combined to form the monodromy matrix. This method is more accurate than solving the monodromy matrix directly. The LMA can not only reduce the reliance on the initial value but also obtain the corresponding monodromy matrix simultaneously in the last iteration. The Floquet multipliers can thus be solved and used to analyze the stability. 


\subsection{Location and stability analysis of the limit cycle}

For a typical continuous-time smooth dynamic system,

$$
\dot{x}=f(x), x \in R^{n},
$$

following assumptions are introduced.

Assumption 1 ([36]) In $f(x)$, for any $x_{1}, x_{2} \in[a, b]$, a strictly positive constant $L$ exists such that,

$$
\left\|f\left(x_{2}\right)-f\left(x_{1}\right)\right\| \leq L\left\|x_{2}-x_{1}\right\| .
$$

Assumption 2 An isolated periodic orbit $L_{0}$ exists in the system and it is called the limit cycle in the phase plane. This cycle is invariant: any trajectory starting on the cycle remains there all the time.

If the above assumptions are satisfied, the equilibrium state of Eq. (31) is no longer an equilibrium point, but an equilibrium limit cycle and its stability determines the stability of the system.

Assume the periodic solution is $L_{0}$ and a point $x_{0} \in L_{0}$. An $n-1$ dimensional smooth hyperplane $\mathrm{S}$ is introduced.

Remark 1 ([37]) This hyperplane is the zero-level set of the smooth scalar function $g$ in the vicinity of $x_{0}$,

$$
\mathrm{S}=\left\{x \in R^{n}: g(x)=0\right\} .
$$

And this hyperplane intersects $L_{0}$ at a nonzero angle, which means that the gradient

$$
\nabla g(x)=\left(\frac{\partial g(x)}{\partial x_{1}}, \frac{\partial g(x)}{\partial x_{2}}, \ldots, \frac{\partial g(x)}{\partial x_{n}}\right)^{T}
$$

is not orthogonal to $L_{0}$ at $x_{0}$, that is, $\left\langle\nabla g\left(x_{0}\right), f\left(x_{0}\right)\right\rangle \neq 0$ where $\langle\cdot, \cdot\rangle$ is the standard scalar product in $R^{n}$.

Definition 1 ([38]) Assume the limit cycle $L_{0}$ of Eq. (31) starts at a point $x_{0}$ on $\mathrm{S}$ and returns to $\mathrm{S}$ at the same point. Besides, an orbit starting at a point $x \in \mathrm{S}$ sufficiently close to $x_{0}$ also returns to $\mathrm{S}$ at some point $\tilde{x} \in \mathrm{S}$ near $x_{0}$. Moreover, nearby orbits will also intersect $\mathrm{S}$ transversally. Thus, a map $P: \mathrm{S} \rightarrow \mathrm{S}$,

$$
x \mapsto \tilde{x}=P(x),
$$

is constructed and this map is called a Poincaré map associated with the cycle $L_{0}$ (shown in Fig. 4). 
After the introduction of the Poincaré map, the existence of the limit cycle $L_{0}$ equals to the existence of the fixed point $x_{0}$ and the stability of the limit cycle $L_{0}$ is now rephrased as the stability of the fixed point $x_{0}$.

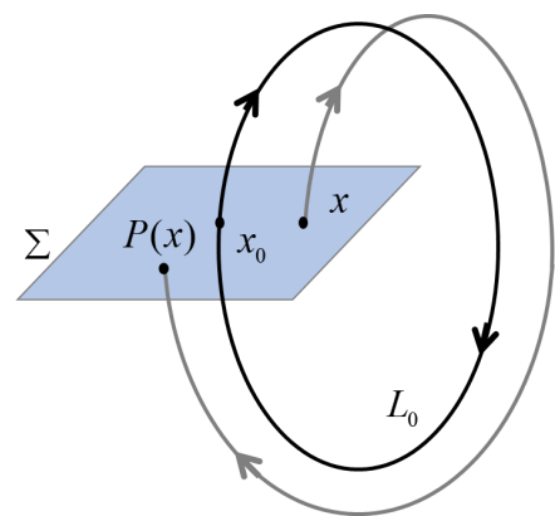

Fig. 4 The Poincaré map associated with the cycle.

Lemma 1 ([37]) The $(n-1) \times(n-1)$ Jacobian matrix of the Poincaré map P taken at the point $x_{0}$ is defined as,

$$
A=\left.\frac{d P}{d x}\right|_{x=x_{0}} .
$$

Its eigenvalues are called characteristic multipliers. If the $n-1$ multipliers have complex modulus less than one, the fixed point is stable.

Remark 2 ([37]) The characteristic multipliers are independent of the hyperplane choice for the Poincare map and the fixed point, because they are a property of the limit cycle.

We further extend Lemma 1 from an autonomous system Eq. (31) to a periodically time-varying non-autonomous system Eq. (30) and give the following theorem.

Theorem 1 For a periodically time-varying system Eq. (30), $x \in R^{n}$ can be used as the coordinates of the hyperplane and the Poincaré map. If the corresponding $n$ multipliers have complex modulus less than one, the fixed point is stable.

Proof The periodically time-varying non-autonomous system Eq. (30) can be transformed into a $n+1$ dimensional periodic autonomous system,

$$
\left\{\begin{array}{l}
\dot{x}=f(\alpha, x) \\
\dot{\alpha}=1, \alpha(0)=0
\end{array}\right.
$$

with coordinates $(\alpha, x) \in S^{1} \times R^{n} \quad\left(\alpha=t(\bmod ) T_{0}\right)$.

After the transformation, Eq. (37) now has $n+1$ dimensional periodic solutions $(\alpha, x)$ and the corresponding hyperplane is $n$ dimensional. Remark 2 presents that the 
selection of the hyperplane is not unique and a natural choice for Eq. (37) is to follow the time cycle $\alpha$,

$$
\mathrm{S}=\{(\alpha, x) \in X: \alpha=0\} .
$$

$x \in R^{n}$ is thus the coordinate on $\mathrm{S}$. Assuming that a solution $x\left(\alpha, x_{0}\right)$ of Eq. (37) exists on the interval $\alpha \in\left[0, T_{0}\right]$ with initial value $x_{0}$, following Poincaré map can be introduced,

$$
x_{0} \mapsto P\left(x_{0}\right)=x\left(T_{0}, x_{0}\right) .
$$

The dimension of the corresponding Jacobian matrix of the Poincare map $P$ in the periodically time-varying system is consequently $n \times n$ and thus has $n$ multipliers. From Lemma 1, we can conclude that if the corresponding $n$ multipliers have complex modulus less than one, the fixed point is stable. Hence Theorem 1 holds.

The next problem to be addressed is how to solve the characteristic multipliers of the original periodically time-varying system Eq. (30).

A linear periodically time-varying system is introduced

$$
\dot{z}=A(t) z \text {. }
$$

Definition 2 ([39]). $\mathrm{F}(t, 0)$ is called the fundamental state transition matrix solution of Eq. (40) from 0 to $t$ if it satisfies

with the initial condition $\mathrm{F}(0,0)=I_{n}$.

$$
\dot{\mathrm{F}}(t, 0)=A(t) \mathrm{F}(t, 0)
$$

Definition 3 ([39]). $\mathrm{F}(T, 0)$ is called a monodromy matrix of the cycle $L_{0}$ if any solution $z(t)$ to Eq. (40) satisfies

$$
z(T)=\mathrm{F}(T, 0) z(0)
$$

Definition 4 ([39]). The eigenvalues $\lambda_{1}, \lambda_{2}, \ldots, \lambda_{n}$ of $\mathrm{F}(T, 0)$ are called Floquet multipliers.

Lemma 2 ([37]) The variation equation is the linear part of the periodically timevarying system governing the evolution of perturbations near the cycle. Both systems share the same properties locally around the limit cycle.

Base on the above, the key theorem on determining the stability of the periodically time-varying system Eq. (30) is presented.

Theorem 2 For a periodically time-varying system Eq. (30) with a limit cycle $L_{0}$, if all Floquet multipliers of the variation equation corresponding to the cycle have complex modulus less than one, the limit cycle is stable. 
Proof Let $x=\varphi(t)$ denotes the periodic solution to the system Eq. (30), $\varphi(t)=\varphi(t+T)$. It is also called a limit cycle $L_{0}$ in the phase plane. Represent a solution to Eq. (30) in the form

$$
x=\xi+\varphi(t),
$$

where $\xi$ is a deviation from the periodic solution. Then, the system can be rewritten as,

$$
\dot{\xi}=P(t) \xi+O\left(\|\xi\|^{2}\right)
$$

where $P(t)=\left.\frac{\partial f}{\partial x}\right|_{x=\varphi(t)}$. By omitting higher terms, a linear periodic system can be obtained

$$
\dot{\xi}=P(t) \xi \text {. }
$$

Eq. (45) is obviously the variational equation of the cycle $L_{0}$. According to Lemma. 2 , it is a linear approximation of the cycle.

The original periodically time-varying system Eq. (30) has been reduced to a linear periodically time-varying system Eq. (45) locally around the cycle. Hence, Floquet theory, an effective method in analyzing the stability of the linear periodically time-varying system, is introduced to evaluate the stability issues of Eq. (45).

From the definition of the state transition matrix in Definition. 2, we can find that it describes a submap from one state to another. More interestingly, the monodromy matrix in Definition 3 describes the map of one whole period, and thus is an option of the Poincaré map specified in Theorem 1. Therefore, the Floquet multipliers of $\mathrm{F}(T, 0)$ coincide with the multipliers of the Poincaré map $P$. According to Theorem. 1, if the Floquet multipliers have complex modulus less than one, the fixed point is stable and the corresponding limit cycle is stable. Hence, Theorem 2 holds.

The monodromy matrix of the cycle $L_{0}$ can be obtained only after exactly locating the limit cycle. The location of the limit cycle is apparently more complicated than that of the equilibrium point, which also hinders the relevant research. Fortunately, this problem can be properly solved by numerical integration. If the initial point for the integration is in the basin of attraction to $L_{0}$, the integration trajectory will finally converge to $L_{0}$. Therefore, the problem of cycle location can be formulated as a Boundary-Value Problem (BVP) and two characters of Eq. (30) facilitate the BVP solving. Firstly, the cycle period T is known in advance and equals 
the flapping period. Secondly, the initial state value can be properly estimated to accelerate the iteration process since these values have practical physical properties and are confined to a certain range. For example, the pitching attitude velocity of Beihawk would not exceed $10 \mathrm{rad} / \mathrm{s}$.

A shooting method has been proved to be an effective method in solving the BVP. However, the simple shooting method can only locate two points on the cycle, the initial and the terminal point, and the obtained monodromy matrix also has an intolerable error. In this paper, a multiple shooting method is introduced to locate any number of points on the cycle. The state transition matrix between the adjacent points is calculated and all these transition matrices can be combined to form the corresponding monodromy matrix after obtaining the exact points position. It is evident that this division can significantly improve the accuracy of the solution by depicting a more detailed cycle.

The multiple shooting method is implemented as follows. The circle is divided into $m$ non-empty subintervals $0=\tau_{0}<\tau_{1}<\ldots<\tau_{m}=T$ and the corresponding coordinate position of the points on the circle are respectively $x^{0}, x^{1}, \ldots, x^{m-1}, x^{m}, x^{j} \in R^{n}$ (shown in Fig. 5). Note that the point $x^{j}$ is composed of several states $\left[x_{1}^{j}, x_{2}^{j}, \ldots, x_{n}^{j}\right]^{T}$. The time intervals are defined as $\Delta_{j}=\tau_{j+1}-\tau_{j}, j=0,1, \ldots, m-1$ and $\left.f\left(x^{j}\right)\right|_{\tau_{j}} ^{\tau_{j+1}}$ denotes the integral results from $\tau_{j}$ to $\tau_{j+1}$ with the initial point value $x^{j}$. Thus, if the following conditions satisfy,

$$
\left\{\begin{array}{l}
\left.f\left(x^{0}\right)\right|_{\tau_{0}} ^{\tau_{1}}-x^{1}=0 \\
\left.f\left(x^{1}\right)\right|_{\tau_{1}} ^{\tau_{2}}-x^{2}=0 \\
\cdots \cdots \\
\left.f\left(x^{m-1}\right)\right|_{\tau_{N-1}} ^{\tau_{N}}-x^{m}=0 \\
x^{m}-x^{0}=0
\end{array} .\right.
$$

$x=\left[x^{0}, x^{1}, \cdots, x^{m-1}, x^{m}\right]^{T}, x \in R^{(m+1) \curvearrowleft n}$ is connected to form the limit cycle as shown in Fig. 5. The Poincaré map is also divided into several submaps correspondingly based on its semigroup property [40]

$$
P=P_{m-1} \square . . \square P_{1} \square P_{0} .
$$

where $P_{j}: \mathrm{S}_{j} \rightarrow \mathrm{S}_{j+1}, j=0,1,2, \ldots, m-1$, and $\mathrm{S}_{j}$ is the hyperplane on the point $x^{j}$.

The fundamental state transition matrix can be selected as a Poincaré map (See the 
proof of Theorem 2) and the matrix also shares the semigroup property,

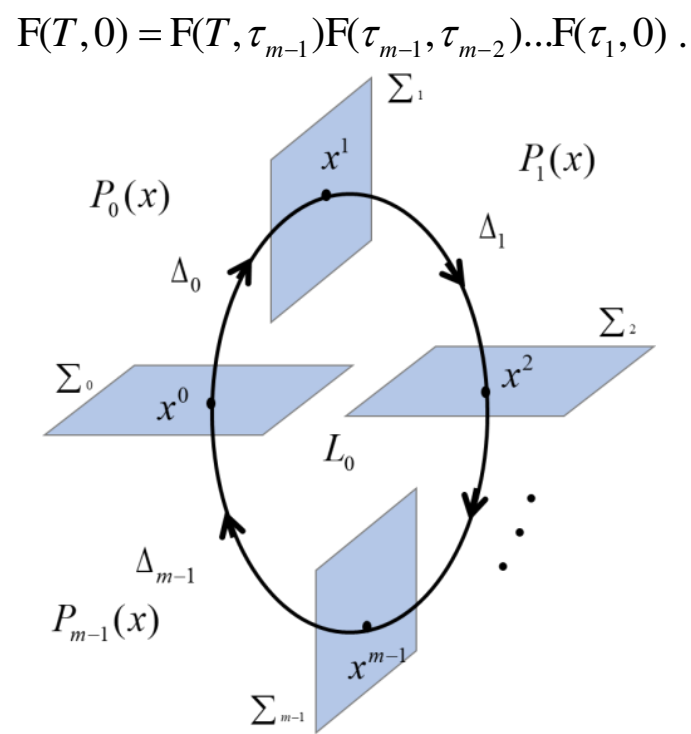

Fig. 5 Locate several points on the limit cycle by multiple shooting method

The multiple shooting problem in this study can be formulated as finding the points $\hat{x}=\left[\hat{x}^{0}, \hat{x}^{1}, \cdots, \hat{x}^{m-1}, \hat{x}^{m}\right], \hat{x} \in R^{(m+1) \llbracket n} \quad$ that $\quad$ can $\quad$ better fit the points $x=\left[x^{0}, x^{1}, \cdots, x^{m-1}, x^{m}\right]$ on the cycle. The concrete computation process is implemented as follows.

The error vector is firstly defined as

$$
\left\{\begin{array}{l}
\left.f\left(\hat{x}^{0}\right)\right|_{\tau_{0}} ^{\tau_{1}}-\hat{x}^{1}=e_{0} \\
\left.f\left(\hat{x}^{1}\right)\right|_{\tau_{1}} ^{\tau_{2}}-\hat{x}^{2}=e_{1} \\
\cdots \cdots \\
\left.f\left(\hat{x}^{m-1}\right)\right|_{\tau_{m-1}} ^{\tau_{m}}-\hat{x}^{m}=e_{m-1} \\
\hat{x}^{m}-\hat{x}^{0}=e_{m}
\end{array} .\right.
$$

For convenience, Eq. (49) is rearranged by defining the left side as the target error function $g=\left[\begin{array}{llll}g_{0} & g_{1} & \cdots & g_{m}\end{array}\right]^{T}, g \in R^{(m+1) \llbracket n}, g_{j} \in R^{n}$ and the right side as the error vector $e=\left[\begin{array}{llll}e_{0} & e_{1} & \cdots & e_{m}\end{array}\right]^{T}, e \in R^{(m+1) \complement n}, e_{j} \in R^{n}$,

$$
g(\hat{x})=e .
$$

The target is now transformed into minimizing the sum of the squares of the errors, i.e., to minimize the quantity

$$
\chi^{2}(\hat{x})=\frac{1}{2} \sum_{j=0}^{m}\left\|g_{j}(\hat{x})\right\|^{2},
$$


where $|\|| \mid$ denotes the 2-norms of the vector.

There are two typical methods for this non-linear least-squares problems: the Gradient Descent and Gauss-Newton methods [41]. The utilized LMA [41] can be regarded as a combination of these two methods and its implementation in a multiple shooting method can significantly improve the robustness of the scheme with respect to the initial value.

The LMA solves the optimization problem by iteration: initiated at the starting point $\hat{x}_{0}$, the method produces a series of solutions $\hat{x}_{1}, \hat{x}_{2}, \ldots$ until the error condition is satisfied. The required Jacobian matrix in the LMA is defined as

$$
J=\left[\begin{array}{ccccc}
\frac{\left.\partial f\left(\hat{x}^{0}\right)\right|_{\tau_{0}} ^{\tau_{1}}}{\partial \hat{x}^{0}} & -I & 0 & \ldots & 0 \\
0 & \frac{\left.\partial f\left(\hat{x}^{1}\right)\right|_{\tau_{1}} ^{\tau_{2}}}{\partial \hat{x}^{1}} & -I & \ldots & 0 \\
\vdots & \vdots & \ddots & \ddots & \vdots \\
0 & 0 & \ldots & \frac{\left.\partial f\left(\hat{x}^{m-1}\right)\right|_{\tau_{m-1}} ^{\tau_{m}}}{\partial \hat{x}^{m-1}} & -I \\
-I & 0 & \ldots & 0 & I
\end{array}\right],
$$

where $\frac{\left.\partial f\left(\hat{x}^{j}\right)\right|_{\tau_{j}} ^{\tau_{j+1}}}{\partial \hat{x}^{j}} \in R^{n \times n}, j=0,1, \ldots, m-1$ and is computed by a numerical method

$$
\frac{\left.\partial f\left(\hat{x}^{j}\right)\right|_{\tau_{j}} ^{\tau_{j+1}}}{\partial \hat{x}^{j}}=\frac{\left.f\left(\hat{x}^{j}+\varepsilon \hat{e}\right)\right|_{\tau_{j}} ^{\tau_{j+1}}-\left.f\left(\hat{x}^{j}\right)\right|_{\tau_{j}} ^{\tau_{j+1}}}{\varepsilon},
$$

where $\varepsilon$ is the differentiation step and $\hat{e}$ is the unit vector.

The advantage of the LMA is its state updating policy and the state augmentation $\delta \hat{x}$ is calculated as follows,

$$
\left[J^{T} J+\lambda I\right] \delta \hat{x}=J^{T} e,
$$

where $\lambda$ is a non-negative, adaptive damping parameter. When the current solution is far from the correct one, $\lambda$ is large and the LMA behaves like a Gradient Descent: slow, but guarantees to converge. When the current solution is close to the correct solution, $\lambda$ is small and the LMA behaves like a Gauss-Newton [42]. Detailed calculation process is presented in the Appendix.

When the trajectory eventually converges on the limit cycle, the norm of the error vector is approximately zero. Thus, 


$$
\frac{\left.\partial f\left(\hat{x}^{j}\right)\right|_{\tau_{j}} ^{\tau_{j+1}}}{\partial \hat{x}^{j}}=\frac{\partial \hat{x}^{j+1}}{\partial \hat{x}^{j}}=\mathrm{F}\left(\tau_{j+1}, \tau_{j}\right) .
$$

The monodromy matrix of the whole cycle can be obtained based on Eq. (48),

$$
\mathrm{F}(T, 0)=\frac{\left.\partial f\left(\hat{x}^{m-1}\right)\right|_{\tau_{m-1}} ^{\tau_{m}}}{\partial \hat{x}^{m-1}} \frac{\left.\partial f\left(\hat{x}^{m-2}\right)\right|_{\tau_{m-2}} ^{\tau_{m-2}}}{\partial \hat{x}^{m-2}} \square . . \frac{\left.\partial f\left(\hat{x}^{0}\right)\right|_{\tau_{0}} ^{\tau_{1}}}{\partial \hat{x}^{0}} .
$$

All of the elements in Eq. (56) can be found in Eq. (52), which presents that the monodromy matrix does not need to be solved separately but is obtained naturally after the location of the limit cycle. The eigenvalues of the monodromy matrix can then be solved and applied to the stability analysis of the system.

\subsection{Stability analysis results of the designed controllers}

The stability of the designed controllers in Section 3.2 is analyzed by the limit cycle location and stability analysis method proposed in Section 4.1.

The SFC is firstly analyzed and the located limit cycle is shown in Fig. 6. The triangles in red are points located by the proposed method and the blue curve describes the practical limit cycle by the numerical integration of the practical system dynamics. It is evident that all points are on the cycle and thus the method can give a precise location result. There is no doubt that an accurate limit cycle location result contributes to the solution of the monodromy matrix and the derived multipliers.

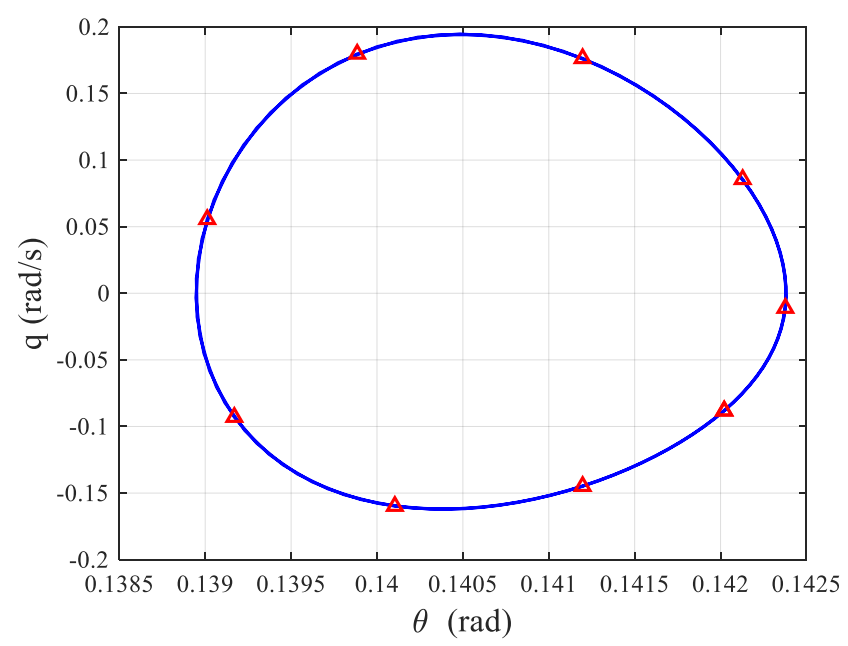

Fig. 6 The located points and the practical limit cycle under the SFC (Triangles in red are the located points and blue curve describes the practical limit cycle)

The corresponding calculated Floquet multipliers (circles in red) are plotted in Fig. 7. These multipliers are inside the unit cycle, and thus the designed SFC is stable. A grid of constant damping factors from zero to one in steps of 0.2 and natural 
frequencies from zero to $\pi$ in step of $\pi / 5$ is also plotted over the current axis. It can be seen that the damping factor of the system is significantly improved compared with the open loop and the aircraft can maintain a more stable hover flight. Here we mainly focus on whether the controller is stable or not. Meanwhile, the dynamic characteristics can be further improved by adjusting related parameters of the aircraft and the controller with the grid graph of the multipliers in Fig. 7.

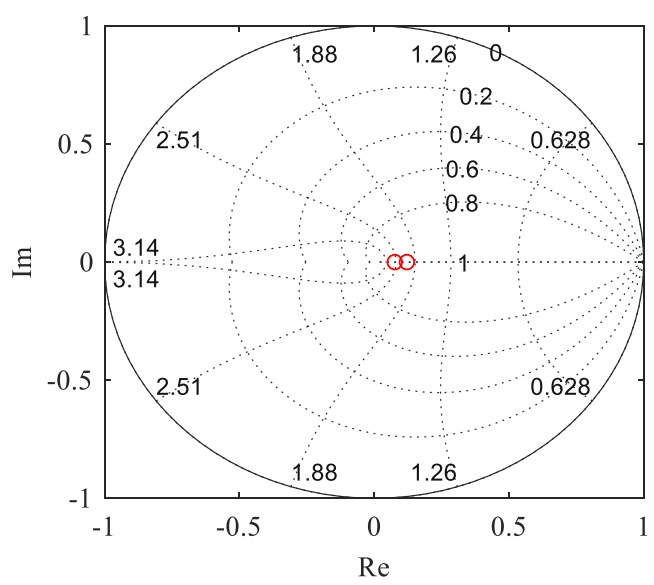

Fig. 7 The position of the Floquet multipliers under the SFC in z-plane

To further verify the stability analysis result by the proposed theorems, we separately choose a random point inside the limit cycle and a random point outside the limit cycle as the initial states and analyze their practical dynamic evolution. Their convergent trajectories are shown in Fig. 8 and the located limit cycle in Fig. 6 is replotted as well. Obviously both the inside one and the outside one converge to the limit cycle. Therefore, the located limit cycle is stable and the proposed method is feasible.

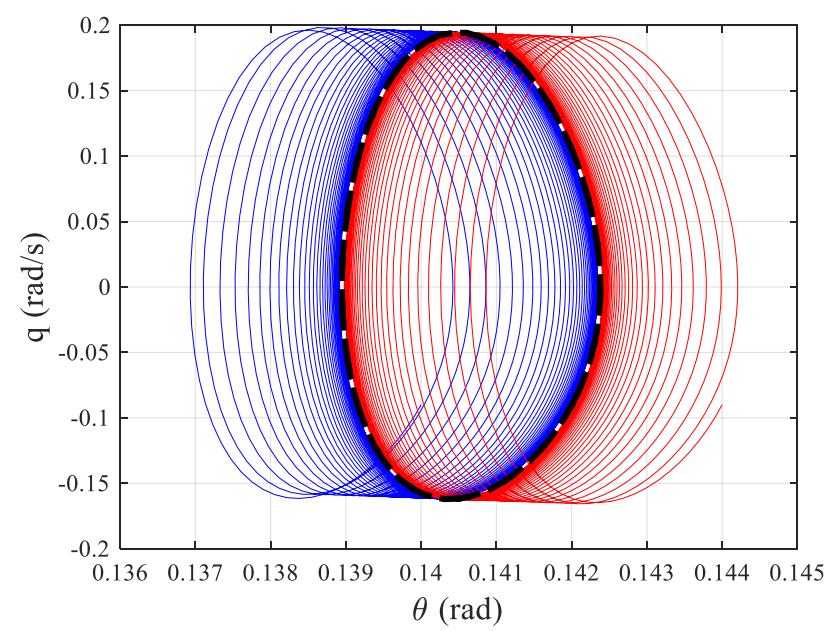

Fig. 8 The convergent trajectories of the point inside the limit cycle (in blue) and outside the limit cycle (in red), and the located limit cycle (dash line in black) under the SFC 
In the stability analysis of the ADRC, the stability issues of the ESO should also be included. The ADRC including the ESO can be presented as follows,

$$
\begin{aligned}
& \dot{x}_{1}=x_{2} \\
& \dot{x}_{2}=d(t)+b(t)\left[k_{1}\left(x_{1 d}-x_{1}\right)+k_{2}\left(x_{2 d}-\hat{x}_{2}\right)-\frac{1}{b} \hat{x}_{3}\right] \\
& \dot{\hat{x}}_{1}=\hat{x}_{2}+l_{1}\left(x_{1}-\hat{x}_{1}\right) \\
& \dot{\hat{x}}_{2}=\hat{x}_{3}+l_{2}\left(x_{1}-\hat{x}_{1}\right)+b\left[k_{1}\left(x_{1 d}-x_{1}\right)+k_{2}\left(x_{2 d}-\hat{x}_{2}\right)-\frac{1}{b} \hat{x}_{3}\right] \\
& \dot{\hat{x}}_{3}=l_{3}\left(x_{1}-\hat{x}_{1}\right)
\end{aligned}
$$

This system has five states including the practical states and the observed states. In this method, the observer and the controller can be easily listed together and analyze the stability of the whole system. This also presents the advantage of our method: the stability issues of any controllers which can be expressed as a periodically timevarying differential system like Eq. (30) can be analyzed via this method.

These overall five states in Eq. (57) cannot be plotted in one coordinate system. Thus, the practical states and the observed states are separately plotted in two coordinate systems shown in Fig. 9 and Fig. 10. The results also indicate that this method can give an accurate location of the limit cycle.

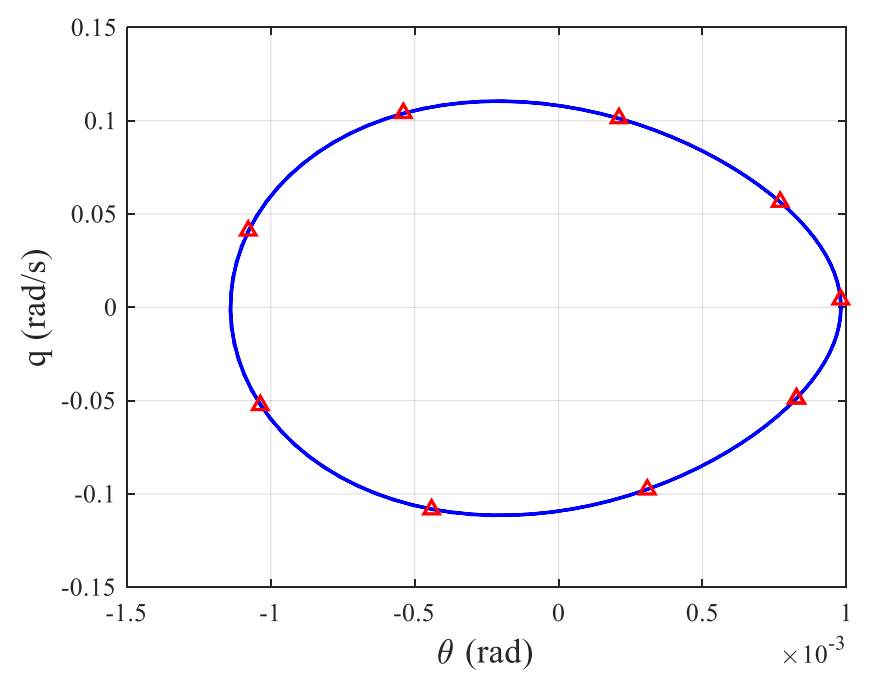

Fig. 9 The located points and the limit cycle of the practical states in ADRC (Triangles in red are the located points and blue curve describes the practical limit cycle) 


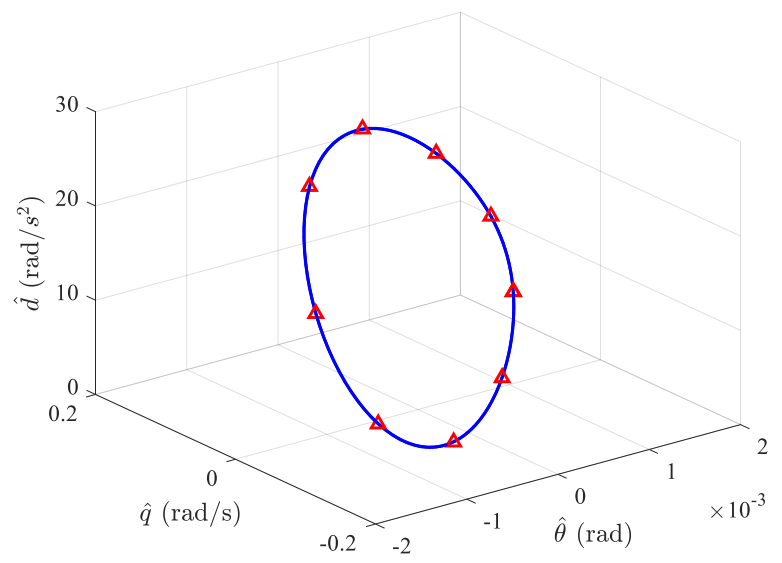

Fig. 10 The located points and the limit cycle of the observed states in ADRC (Triangles in red are the located points and blue curve describes the practical limit cycle)

The corresponding Floquet multipliers (circles in red) are also plotted in Fig. 11. The five multipliers inside the unit cycle also prove the stability of the ADRC.

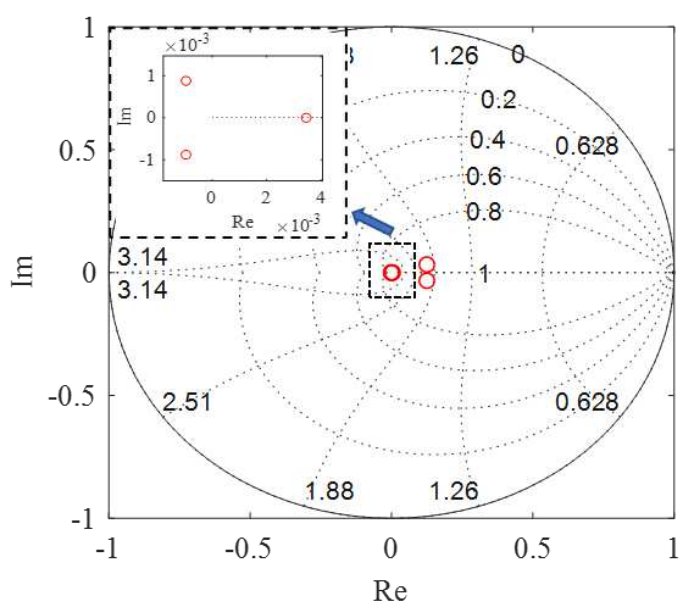

Fig. 11 The position of the Floquet multipliers under the ADRC in z-plane

The stability analysis result of the ADRC is also tested and the corresponding dynamic evolutions are shown in Fig. 12 and Fig. 13. The trajectories on both sides of the limit cycle converge on the limit cycle, which also confirms the stability analysis method proposed in this paper. 


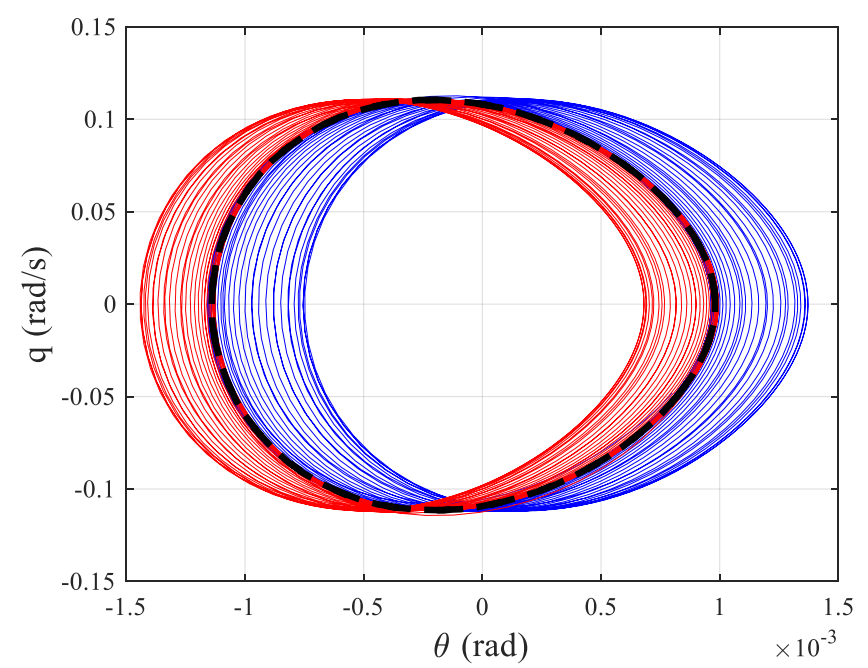

Fig. 12 The convergent trajectories of the point inside the limit cycle (in blue) and outside the limit cycle (in red), and the located limit cycle (dash line in black) of the practical states in ADRC

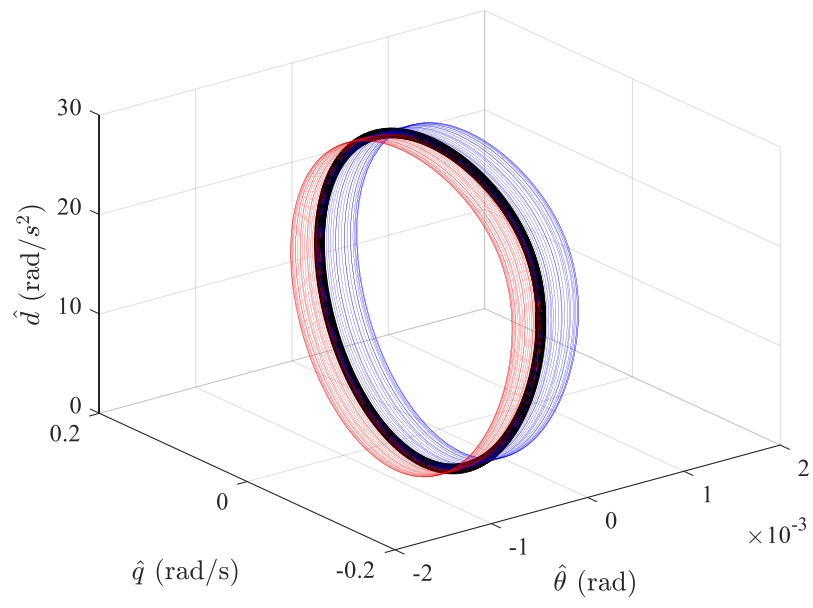

Fig. 13 The convergent trajectories of the point inside the limit cycle (in blue) and outside the limit cycle (in red), and the located limit cycle (in black) of the observed states in ADRC

In this section, the proposed method is utilized to analyze the stability of the periodically time-varying flapping-wing aircraft under the SFC and the ADRC without the prior assumption of the averaging theory. The analysis results are validated by simulation and proved to be accurate. Thus, the controller design and stability analysis in flapping-wing aircraft is no longer limited by the range of flapping frequency. This method can also give a more detailed description of the timevarying features in flapping-wing aircraft, like the interesting limit cycle oscillation, which cannot be observed in the averaged system. 


\section{Flight control results}

After ensuring the stability of the designed controllers, they are separately used to control Beihawk in hover. Relevant descriptions of the experimental platform have been presented in the previous work [6]. The longitudinal dynamics is the most challenging part in the hover control. Therefore, we mainly focus on the pitching attitude control effect in this work and the control results are shown as follows.

\subsection{State feedback controller}

The parameters of the SFC are set to be $k_{p}=2$ and $k_{d}=1$ to obtain a satisfactory control effects. The desired pitching attitude and attitude velocity are set to be zero to stay hover status and the control effects are shown in Fig. 14.
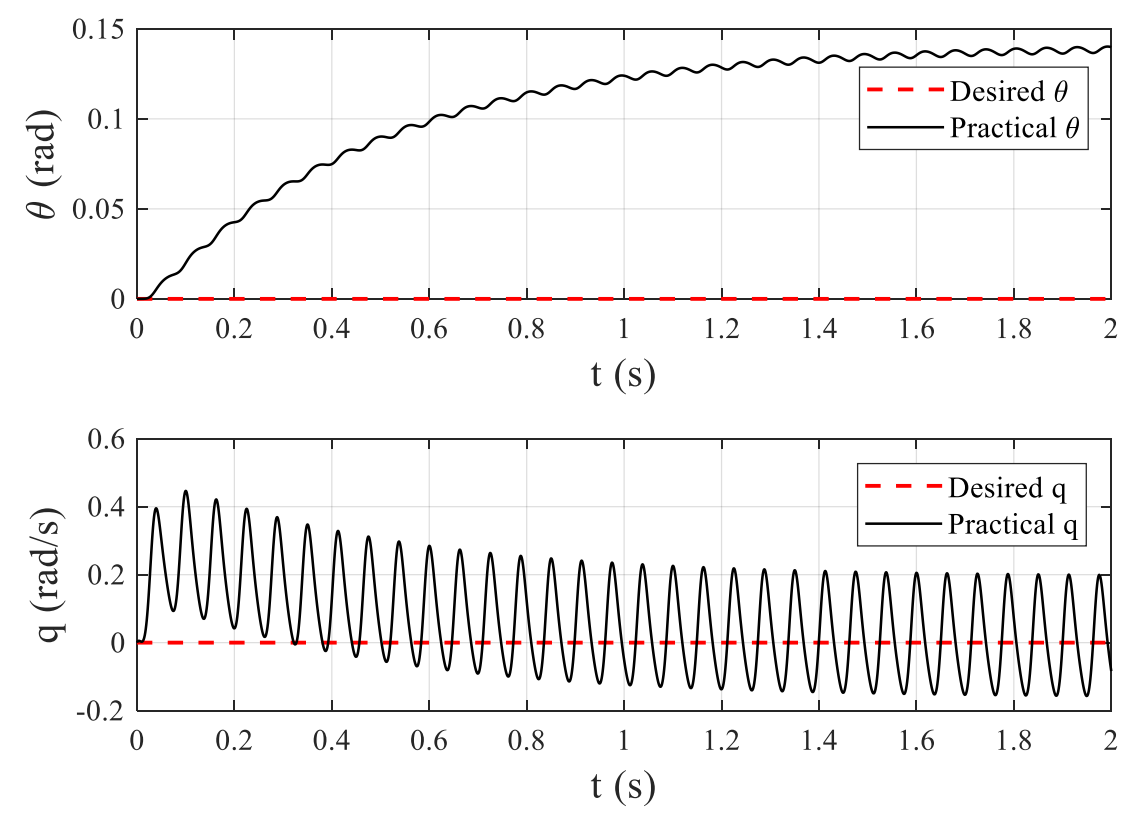

Fig. 14 Pitching attitude control effect of the SFC

It can be seen that the SFC can only achieve the hover stability augmentation of Beihawk. Large attitude oscillations still exist in both the pitching attitude and pitching attitude velocity in one flapping period. Due to the disturbing torque generated by the flapping motion, the pitching attitude cannot reach the desired value. The tail control mechanism under the SFC cannot completely counteract the attitude response error and it is necessary to apply advanced controller. 


\subsection{Active disturbance rejection controller}

The parameters $k_{1}$ and $k_{2}$ of the ADRC are designed the same as the SFC to highlight the superiority of the ESO in the ADRC. The parameters of the ESO are designed according to the bandwidth parameterization results in [43]:

$$
\left\{\begin{array}{l}
l_{1}=3 \omega_{0} \\
l_{2}=3 \omega_{0}^{2} \\
l_{1}=\omega_{0}^{3}
\end{array},\right.
$$

where $\omega_{0}$ is the observer bandwidth. Here $\omega_{0}$ is designed to be 1000 considering the maximum $10 \mathrm{~Hz}$ flapping frequency. The control effects and the observed results are shown in Fig. 15, 16.
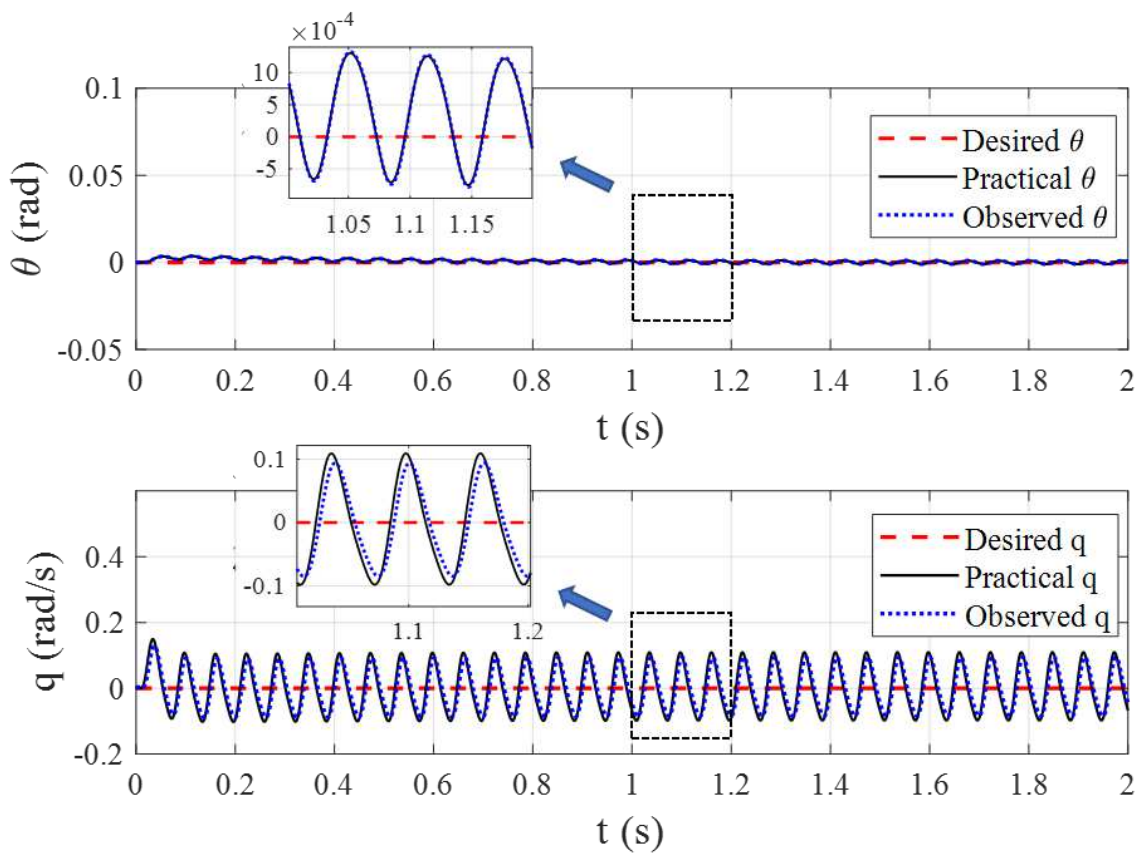

Fig. 15 Control effects and states observation results of the pitching attitude under the ADRC

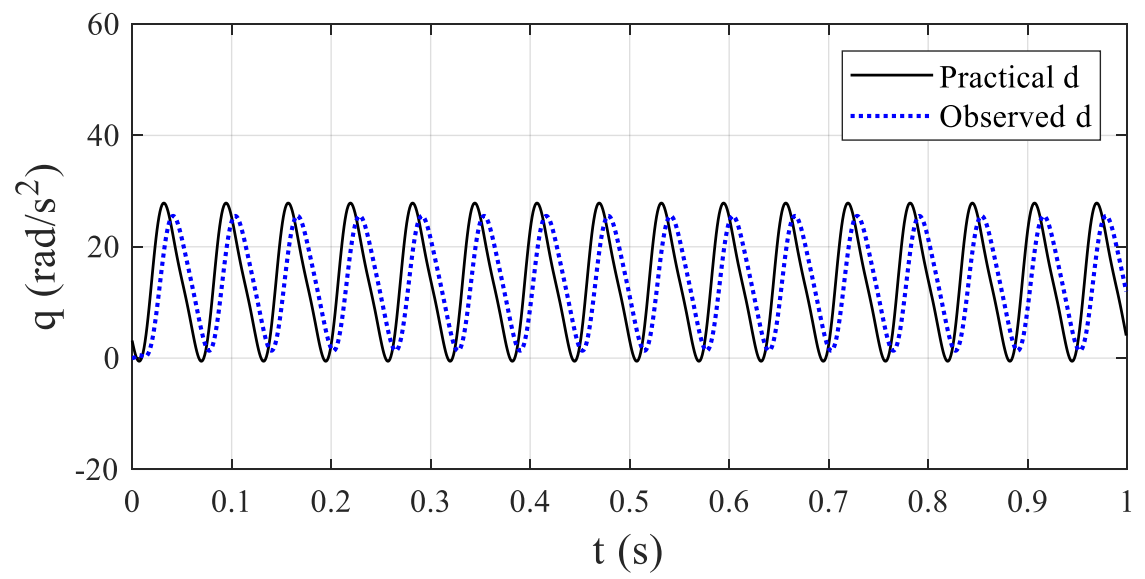

Fig. 16 Disturbance observation result under the ADRC 
It can be seen that the ESO can not only accurately observe the system state but also give a satisfactory observation of the disturbance although the varying frequency of the generated disturbance is considerably high, the same as the flapping frequency. Besides, the ADRC can effectively compensate the attitude response errors and the compensated attitude fluctuates periodically around the desired pitching attitude. Finally, as we all know, the limit cycle oscillation is the inherent feature of flappingwing aircraft. It cannot be entirely counteracted and can only be suppressed. The ADRC can better suppress the oscillation amplitude than the SFC. The Mean Value (MV) and Root Mean Square (RMS) of the error under both controllers are separately calculated as listed in Table 2 and the results quantitively confirm the above conclusions.

Table 2 Comparison between the two controllers

\begin{tabular}{c|c|cc}
\hline \multicolumn{2}{c|}{ Controller } & $\begin{array}{c}\text { Feedback } \\
\text { Controller }\end{array}$ & $\begin{array}{c}\text { ADRC } \\
\text { Controller }\end{array}$ \\
\hline $\begin{array}{c}\text { Error } \\
\text { Mean } \\
\text { value }\end{array}$ & $\theta$ & $1.06 \mathrm{e}-01$ & $8.45 \mathrm{e}-04$ \\
\cline { 2 - 4 } $\begin{array}{c}\text { Root mean } \\
\text { square }\end{array}$ & $q$ & $6.98-02$ & $3.18 \mathrm{e}-04$ \\
\cline { 2 - 4 } & $q$ & $1.40 \mathrm{e}-03$ & $1.38 \mathrm{e}-06$ \\
\hline
\end{tabular}

Both the SFC and the ADRC can achieve the stability augmentation of the flapping-wing aircraft, Beihawk, in hover. The ADRC performs better in attitude limit cycle oscillation suppression than the SFC. Because it can make full use of the built wing-tail interaction model to observe the disturbance with the aid of the ESO and compensate the observed disturbance in the control signal design process. This also emphasizes the importance of an accurate tail model in the attitude controller design.

\section{Conclusion}

The widely-used averaging theory fails to reveal the period limit cycle properties in flapping flight and may lead to erroneous results on the stability analysis. In this study, the critical periodically time-varying feature of the flapping-wing aircraft Beihawk is considered in dynamic modelling, controller design and stability analysis. This consideration facilitates the unique limit cycle oscillation study in flapping flight. To present an easily implemented periodically time-varying model, DFT is applied to the original model and the transformed model is found to be of 96 percent fidelity by reserving the harmonics less than quintuple flapping frequency. 
Based on this model, two controllers are separately designed to suppress the inherent oscillation. The stability of the controller is analyzed in terms of the unique equilibrium limit cycle which is apparently more complex than the equilibrium point. Poincaré map is introduced to simplify this cycle to an equivalent fixed point. A multiple shooting method is employed to locate several points on the cycle and calculate the submaps between the adjacent points. All these submaps are combined to form the Poincaré map and this division markedly improves the accuracy of the solved whole map. The characteristic multipliers associated with the Poincaré map can give an estimation of the fixed point's stability. The located points are discovered to be exactly distributed on the practical limit cycle solved by the numerical integration of the dynamics. The stability analysis results characterized by the multipliers coincide with the practical dynamic evolution represented by the convergent trajectories inside and outside the cycle. The proposed method can accurately describe the periodic feature and the stability of flapping flight and relevant conclusions are no longer constrained by the range of flapping frequency.

The designed controllers are used to control Beihawk after ensuring their stability. Both controllers can achieve stability augmentation while the ADRC performs better in suppressing the oscillation. This superiority of the ADRC can be attributed to the built wing-tail interaction model which is fully utilized to aid disturbance observation in the ESO and disturbance compensation in the control signal design. This also indicates the importance of tail control mechanism in the attitude stabilization and the necessity of an accurate tail model. The limit cycle oscillation suppression presents powerful research potential after realizing the stability augmentation. It enables Beihawk to achieve a more stable flight and to capture higher resolution images while carrying a camera.

The proposed period-based stability analysis method is of high flexibility and it can be easily extended to analyze the stability of other controllers in flapping flight. Moreover, it has potential applications in analyzing the influence of parameters variation on the damping and frequency features of flight dynamics which is described by the position of the characteristic multipliers in the grid graph.

\section{Acknowledgements}

The authors acknowledge the financial support from the National Natural Science 
Foundation of China under grants 51905014. The authors would also like to thank the editors and reviewers for their critical review of this manuscript.

\section{Data availability}

All data used to support the findings of this study are available from the corresponding author upon request.

\section{Code availability}

This work is done using custom code.

\section{Ethics declarations}

\section{Conflict of interest}

The authors declare that there is no conflict of interest regarding the publication of this paper.

\section{Appendix}

Algorithm description of the LMA: Solving the located points on the circle.

\section{Initialization:}

Indicative values for the user-defined parameters:

$\tau=10^{-3}, \varepsilon_{1}=\varepsilon_{2}=\varepsilon_{3}=10^{-15}, k_{\max }=100$.

Input: A target error function $g(\hat{x})$ and an initial state vector $\hat{x}_{0} \in R^{(m+1) \sqsubset n}$.

Output: A vector $\hat{x}^{+} \in R^{(m+1) \sqsubset n}$ minimizing $\chi^{2}(\hat{x})$.

\section{Algorithm:}

$k:=0 ; v:=2 ; \hat{x}:=\hat{x}_{0} ;$

$A:=J^{T} J ; e=g(\hat{x}) ; h:=J^{T} e ;$

stop: $=\left(\|g\|_{\infty} \leq \varepsilon_{1}\right) ; \lambda=\tau * \max _{i=1, \ldots, m}\left(A_{i i}\right) ;$

while (not stop) and $\left(k<k_{\max }\right)$

$$
k:=k+1
$$

repeat 
Solve $(A+\lambda I) \delta \hat{x}=h$;

$$
\begin{aligned}
& \text { if }\left(\|\delta \hat{x}\| \leq \varepsilon_{2}\|\hat{x}\|\right) \\
& \text { stop }:=\text { true; } \\
& \text { else } \\
& \hat{x}_{\text {new }}:=\hat{x}+\delta \hat{x} ; \\
& \rho:=\left(\|e\|^{2}-\left\|g\left(\hat{x}_{\text {new }}\right)\right\|^{2}\right) /\left(\delta \hat{x}^{T}(\lambda \delta \hat{x}+h)\right) ; \\
& \text { if } \rho>0 \\
& \hat{x}:=\hat{x}_{\text {new }} ; \\
& A:=J^{T} J ; e=g(\hat{x}) ; h:=J^{T} e ; \\
& \text { stop: }=\left(\|h\|_{\infty} \leq \varepsilon_{1}\right) \text { or }\left(\|e\|^{2} \leq \varepsilon_{3}\right) \\
& \lambda:=\lambda * \max \left(\frac{1}{3}, 1-(2 \rho-1)^{3}\right) ; \quad v:=2 ;
\end{aligned}
$$

else

$$
\lambda:=\lambda * v ; \quad v:=2 * v
$$

\section{endif}

\section{endif}

until $(\rho>0)$ or (stop)

\section{endwhile}

$\hat{x}^{+}:=\hat{x}$

\section{References}

1. Hoff, J., Syed, U., Ramezani, A., Hutchinson, S.: Trajectory planning for a bat-like flapping wing robot. IEEE Int. Conf. Intell. Robot. Syst. 6800-6805 (2019). https://doi.org/10.1109/IROS40897.2019.8968450

2. Lee, J., Ryu, S., Kim, H.J.: Stable Flight of a Flapping-Wing Micro Air Vehicle under Wind Disturbance. IEEE Robot. Autom. Lett. 5, 5685-5692 (2020). https://doi.org/10.1109/LRA.2020.3009064

3. Warrick, D.R., Tobalske, B.W., Powers, D.R.: Aerodynamics of the hovering hummingbird. Nature. 435, 1094-1097 (2005). https://doi.org/10.1038/nature03647

4. De Croon, G.C.H.E., Groen, M.A., De Wagter, C., Remes, B., Ruijsink, R., Van Oudheusden, B.W.: Design, aerodynamics and autonomy of the DelFly. Bioinspiration and Biomimetics. 7, (2012). https://doi.org/10.1088/1748-3182/7/2/025003

5. Robert Wood, R.N. and G.-Y.W.S.: Flight of the robobees. Sci. Am. 308, 60-65 (2013) 
6. Jiao, Z., Wang, L., Zhao, L., Jiang, W.: Hover flight control of X-shaped flapping wing aircraft considering wing-tail interactions. Aerosp. Sci. Technol. 116, 106870 (2021). https://doi.org/10.1016/j.ast.2021.106870

7. Banazadeh, A., Taymourtash, N.: Adaptive attitude and position control of an insect-like flapping wing air vehicle. Nonlinear Dyn. 85, 47-66 (2016). https://doi.org/10.1007/s11071016-2666-8

8. Shyy, W., Berg, M., Ljungqvist, D.: Flapping and flexible wings for biological and micro air vehicles. (1999)

9. Sane, S.P.: The aerodynamics of insect flight. J. Exp. Biol. 206, 4191-4208 (2003). https://doi.org/10.1242/jeb.00663

10. Wang, Z.J.: Dissecting insect flight. Annu. Rev. Fluid Mech. 37, 183-210 (2005). https://doi.org/10.1146/annurev.fluid.36.050802.121940

11. Xuan, H., Hu, J., Yu, Y., Zhang, J.: Recent progress in aerodynamic modeling methods for flapping flight. AIP Adv. 10, (2020). https://doi.org/10.1063/1.5130900

12. Terze, Z., Pandža, V., Kasalo, M., Zlatar, D.: Optimized flapping wing dynamics via DMOC approach. Nonlinear Dyn. 103, 399-417 (2021). https://doi.org/10.1007/s11071-020-06119-y

13. Deng, X., Schenato, L., Sastry, S.S.: Flapping Flight for Biomimetic Robotic Insects: Part ISystem Modeling. IEEE Trans. Robot. 22, 776-788 (2006)

14. He, W., Yan, Z., Sun, C., Chen, Y.: Adaptive neural network control of a flapping Wing micro aerial vehicle with disturbance observer. IEEE Trans. Cybern. 47, 3452-3465 (2017). https://doi.org/10.1109/TCYB.2017.2720801

15. Maggia, M., Eisa, S.A., Taha, H.E.: On higher-order averaging of time-periodic systems: reconciliation of two averaging techniques. Nonlinear Dyn. 99, 813-836 (2020). https://doi.org/10.1007/s11071-019-05085-4

16. Deng, X., Schenato, L., Sastry, S.S.: Flapping flight for biomimetic robotic insects: Part II Flight control design. IEEE Trans. Robot. 22, 789-803 (2006). https://doi.org/10.1109/TRO.2006.875483

17. Cheng, B., Deng, X.: A neural adaptive controller in flapping flight. J. Robot. Mechatronics. 24, 602-611 (2012). https://doi.org/10.20965/jrm.2012.p0602

18. Zhang, J., Tu, Z., Fei, F., Deng, X.: Geometric flight control of a hovering robotic hummingbird. Proc. - IEEE Int. Conf. Robot. Autom. 5415-5421 (2017). https://doi.org/10.1109/ICRA.2017.7989638

19. Fei, F., Tu, Z., Zhang, J., Deng, X.: Learning extreme hummingbird maneuvers on flapping wing robots. Proc. - IEEE Int. Conf. Robot. Autom. 2019-May, 109-115 (2019). https://doi.org/10.1109/ICRA.2019.8794100

20. Wissa, B.E., Elshafei, K.O., El-Badawy, A.A.: Lyapunov-based control and trajectory tracking of a 6-DOF flapping wing micro aerial vehicle. Nonlinear Dyn. 99, 2919-2938 (2020). https://doi.org/10.1007/s11071-020-05487-9 
21. Chirarattananon, P., Ma, K.Y., Wood, R.J.: Adaptive control of a millimeter-scale flappingwing robot. Bioinspiration and Biomimetics. 9, (2014). https://doi.org/10.1088/1748$3182 / 9 / 2 / 025004$

22. Taha, H.E., Hajj, M.R., Nayfeh, A.H.: Flight dynamics and control of flapping-wing MAVs: A review. Nonlinear Dyn. 70, 907-939 (2012). https://doi.org/10.1007/s11071-012-0529-5

23. Ducci, G., Colognesi, V., Vitucci, G., Chatelain, P., Ronsse, R.: Stability and Sensitivity Analysis of Bird Flapping Flight. J. Nonlinear Sci. 31, 1-32 (2021). https://doi.org/10.1007/s00332-021-09698-1

24. Su, W., Cesnik, C.E.S.: Flight dynamic stability of a flapping wing Micro Air Vehicle in hover. Collect. Tech. Pap. - AIAA/ASME/ASCE/AHS/ASC Struct. Struct. Dyn. Mater. Conf. 1-17 (2011). https://doi.org/10.2514/6.2011-2009

25. Dietl, J.M., Garcia, E.: Stability in hovering ornithopter flight. Ind. Commer. Appl. Smart Struct. Technol. 2008. 6930, 69300N (2008). https://doi.org/10.1117/12.776482

26. Sane, S.P., Dickinson, M.H.: The aerodynamic effects of wing rotation and a revised quasisteady model of flapping flight. J. Exp. Biol. 205, 1087-1096 (2002)

27. Robyn Lynn Harmon: Aerodynamic modelling of a flapping membrane wing using motion tracking experiments, (2008)

28. Dickinson, M.H., Lehmann, F., Sane, S.P., Dickinson, M.H., Lehmann, F., Sane, S.P.: Wing Rotation and the Aerodynamic Basis of Insect Flight. Science (80-. ). 284, 1954-1960 (1999)

29. Y. Fung: An Introduction to the Theory of Aeroelasticity. Dover, New York (1969)

30. Sane, S.P., Dickinson, M.H.: Erratum: The control of flight force by a flapping wing: Lift and drag production (Journal of Expiremental Biology (2001) 204 (2607-2626)). J. Exp. Biol. 204, $3401(2001)$

31. Anderson, J.D.: Fundamentals of Aerodynamics, Sixth Edition. (2017)

32. Oppermann, R.H.: The elements of aerofoil and airscrew theory, (1943)

33. Han, J.: From PID to active disturbance rejection control. IEEE Trans. Ind. Electron. 56, 900906 (2009). https://doi.org/10.1109/TIE.2008.2011621

34. Madonski, R., Shao, S., Zhang, H., Gao, Z., Yang, J., Li, S.: General error-based active disturbance rejection control for swift industrial implementations. Control Eng. Pract. 84, $218-$ 229 (2019). https://doi.org/10.1016/j.conengprac.2018.11.021

35. Wang, C., Chen, Z., Sun, Q., Qing, Z.: Design of PID and ADRC based quadrotor helicopter control system. Proc. 28th Chinese Control Decis. Conf. CCDC 2016. 5860-5865 (2016). https://doi.org/10.1109/CCDC.2016.7532046

36. HASSAN K. KHALIL: Nonlinear SysteIlls Third Edition. (2002)

37. Kuznetsov, Y.A.: Elements of Applied Bifurcation Theory, Second Edition. 614 (1998)

38. Verhulst, F.: Ferdinand Verhulst Nonlinear Differential Equations and Dynamical Systems. (1989)

39. Amin, M.A., Hertzberg, M.P., Kaiser, D.I., Karouby, J.: Nonperturbative dynamics of reheating after inflation: A review. Int. J. Mod. Phys. D. 24, 1-46 (2015). https://doi.org/10.1142/S0218271815300037 
40. Dietl, J.M., Garcia, E.: Stability in ornithopter longitudinal flight dynamics. J. Guid. Control. Dyn. 31, 1157-1162 (2008). https://doi.org/10.2514/1.33561

41. Dednam, W., Botha, A.E.: Optimized shooting method for finding periodic orbits of nonlinear dynamical systems. Eng. Comput. 31, 749-762 (2015). https://doi.org/10.1007/s00366-0140386-6

42. Lourakis, M.I. a: A Brief Description of the Levenberg-Marquardt Algorithm Implemened by levmar. Matrix. 3, 2 (2005)

43. Gao, Z.: Scaling and Bandwidth-Parameterization based Controller Tuning. Proc. Am. Control Conf. 6, 4989-4996 (2003). https://doi.org/10.1109/acc.2003.1242516 